\title{
The magnetic fine structure of the Sun's polar region as revealed by Sunrise
}

\author{
A. Prabhu ${ }^{1}$, A. $\operatorname{Lagg}^{1}$, J. Hirzberger ${ }^{1}$, and S. K. Solanki ${ }^{1,2}$ \\ 1 Max-Planck-Institut für Sonnensystemforschung, Justus-von-Liebig-Weg 3, 37077 Göttingen, Germany \\ e-mail: prabhu@mps.mpg.de \\ 2 School of Space Research, Kyung Hee University, Yongin, Gyeonggi 446-701, Republic of Korea
}

Received 19 June 2020 / Accepted 29 September 2020

\begin{abstract}
Context. Polar magnetic fields play a key role in the solar magnetic cycle and they are the source of a significant portion of the interplanetary magnetic field. However, observations of the poles are challenging and hence our understanding of the polar magnetic environment is incomplete.

Aims. We deduce properties of small-scale magnetic features in the polar region using high-resolution data and specifically aim to determine the flux per patch above which one magnetic polarity starts to dominate over the other.

Methods. We study the high spatial resolution, seeing-free observations of the north solar polar region, obtained with the IMaX instrument on-board the balloon-borne SUNRISE observatory during June 2009, at the solar activity minimum. We performed inversions of the full Stokes vector recorded by IMaX to retrieve atmospheric parameters of the Sun's polar region, mainly the temperature stratification and the magnetic field vector.

Results. We infer kilo-Gauss (kG) magnetic fields in patches harbouring polar faculae, without resorting to a magnetic filling factor Within these patches we find the maxima of the magnetic field to be near the dark narrow lanes, which are shifted towards the disc centre side in comparison to the maxima in continuum intensity. In contrast, we did not find any fields parallel to the solar surface with $\mathrm{kG}$ strengths. In addition to the $\mathrm{kG}$ patches, we found the polar region to be covered in patches of both polarities, which have a range of sizes. We find the field strength of these patches to increase with increasing size and flux, with the smaller patches showing a significant dispersion in field strength. The dominating polarity of the north pole during this phase of the solar cycle is found to be maintained by the larger patches with fluxes above $2.3 \times 10^{17} \mathrm{Mx}$.
\end{abstract}

Key words. Sun: magnetic fields - Sun: faculae, plages

\section{Introduction}

A thorough and coherent investigation of the Sun's polar magnetic environment is a key element, not only for a better understanding of fundamental processes in the Sun, such as the solar activity cycle, but also for the structure of a significant portion of the interplanetary magnetic field, especially at the minimum of the activity cycle. An inference of the strength, orientation, and spatial distribution of the polar magnetic fields is a valuable predictor of the strength of the subsequent cycle (Schatten et al. 1978; Cameron \& Schüssler 2007, 2015; Wang \& Sheeley 2009). Global and long-term studies of the magnetic activity at the poles, based on low-resolution data or synoptic magnetic field maps, have provided insight into, the polar coronal holes (Wang 2009), polar plumes (Gabriel et al. 2009), the source of the fast solar wind (Hassler et al. 1999; Tu et al. 2005), and the large-scale effect of the solar dynamo, or to compare the total solar irradiance of the Sun with that of other Sun-like stars (see, e.g. Schatten 1993; Knaack et al. 2001) or the influence of inclination variations of Earth's orbit on its climate (Vieira et al. 2012). See Petrie (2015) for a review of polar fields and their influence on the solar atmosphere.

During the last decades, the polar magnetic landscape has also increasingly been studied at the smallest observable spatial scales. For example, the presence of polar faculae harbouring kilo-Gauss $(\mathrm{kG})$ magnetic fields was reported by several authors analysing ground-based observations (Homann et al. 1997; Okunev \& Kneer 2004; Blanco Rodríguez et al. 2007). These studies were based on the inference of the magnetic fields from observations of Stokes $I$ and $V$ of Fraunhofer lines in the visible spectral range. The most comprehensive picture of the magnetic fine structure at the Sun's poles was achieved using the Solar Optical Telescope (SOT, Tsuneta et al. 2008a; Suematsu et al. 2008; Shimizu et al. 2008) on-board the Hinode spacecraft (Kosugi et al. 2007), which enabled access to vector maps of the polar magnetic landscape. Tsuneta et al. (2008b) investigated the data from SOT spectropolarimeter (SOT/SP) and found patches of unipolar magnetic flux concentrations with field strengths exceeding $1 \mathrm{kG}$, wherein the magnetic field vector is nearly vertical to the local solar surface. They reported ubiquitous and relatively weaker horizontal fields (i.e. parallel to the solar surface), with no spatial correlation between the vertical and horizontal fields. Blanco Rodríguez \& Kneer (2010) extended their previous study (Blanco Rodríguez et al. 2007) of the polar faculae, analysing the full Stokes vector in the infrared $\mathrm{Fe}$ I lines at $1.5 \mu \mathrm{m}$. They concluded that the detected polar faculae did not harbour sufficient magnetic flux to account for the global flux inferred from magnetograms (at low spatial resolution) by Svalgaard et al. (1978) or from in situ magnetic field measurements of the solar wind by the Ulysses spacecraft (McComas et al. 2000; Svalgaard et al. 1978). Linker et al. (2017) estimated the interplanetary magnetic flux directly from 
synoptic magnetic maps. They isolate possible causes for the underestimation of this estimated interplanetary magnetic flux and pointed out that one of the possible reasons lies in the underestimation of the polar magnetic flux at the photospheric levels of the Sun.

Ito et al. (2010), using Hinode observations, found the net magnetic flux and average area of the $\mathrm{kG}$ magnetic concentrations in the polar region to be larger than the magnetic field concentrations of the quiet Sun at lower latitudes. In the study of Shiota et al. (2012) regarding the polar field reversals, also with Hinode observations, they report that the large magnetic concentrations in the polar regions harbour flux that is comparable to that of pores. In addition, they conclude that large magnetic patches with a flux $\geq 10^{18} \mathrm{Mx}$ tend to be unipolar, whereas smaller patches with a flux lower than $10^{18} \mathrm{Mx}$ are relatively flux-balanced. Therefore, the overall global polarity of the polar regions is determined exclusively by the large magnetic patches.

Kaithakkal et al. $(2013,2015)$ also analysed Hinode data to study the morphology of large polar magnetic patches and the role of magnetoconvection in the formation and decay of these flux concentrations. Both Shiota et al. (2012) and Kaithakkal et al. (2015) stress that the unipolar appearance and disappearance of the magnetic patches in the Hinode/SOT observations indicates the presence of magnetic flux below the detection limit of the instrument. Quintero Noda et al. (2016) in their study of the polar faculae, performed height stratified inversions of Hinode data and retrieved nearly vertical $\mathrm{kG}$ magnetic fields of a single polarity within polar faculae. They found these magnetic fields spatially shifted towards the disc centre with respect to the corresponding continuum images. More recently, Pastor Yabar et al. (2018) also confirmed the presence of strong vertical fields, and reported polarimetric signals compatible with the presence of unresolved small-scale loops. In a subsequent study, Pastor Yabar et al. (2020) extend their analysis to a larger fraction of the north polar region close to solar maximum. They found the physical properties of the polar region to be in agreement with those of the quiet Sun at disc centre and the low latitude east limb.

Despite the progress achieved especially during the last decade, studies of polar magnetic fields have to fight against some principal problems of spectropolarimetric observations: Image reconstruction techniques employed to counter the effects of atmospheric seeing lower the signal-to-noise ratio of groundbased observations. The space-borne observatory Hinode has insufficient spatial resolution to resolve the small-scale magnetic patches, especially when observed at the poles at high heliocentric angles. This not only results in a strong foreshortening, but also introduces a large intensity gradient when approaching the solar limb. Because of these difficulties, many authors use a simplistic inversion technique based on the non-physical stratification of temperatures in the so-called Milne-Eddington approximation. In this study we report on our analysis of higher resolution, seeing-free observations of the solar polar region acquired by the SUNRISE balloon-borne observatory (Solanki et al. 2010; Barthol et al. 2011; Berkefeld et al. 2011; Gandorfer et al. 2011) during its first flight in 2009. By adopting a novel approach to inverting spectropolarimetric data recorded at high heliocentric angles, we retrieve magnetic field distribution and temperature stratification at a high spatial resolution. Our focus is on the small-scale features. We specifically aim to investigate at what scales and flux values do patches of one polarity dominate over the other, to give the solar poles a unique dominant polarity.

The structure of the paper is as follows. We describe the observations in Sect. 2, the details of our inversions in Sect. 3 and present and discuss our findings in the context of the available literature in Sects. 4 and 5.

\section{Observations and data reduction}

We study data from two consecutive observing runs recorded on the 16 of June 2009 starting 16:20 UT during the first flight of SUNRISE. Here we analyse data from the Imaging Magnetograph eXperiment (IMaX, Martínez Pillet et al. 2011). IMaX recorded the full Stokes vector in the V5-6 observing mode scanning the Fe I 5250.2 $\AA$ line (Landé factor $g=3$ ) at five spectral positions located at $[-80,-40,+40,+80] \mathrm{m} \AA$ (4 positions in the spectral line), and $+227 \mathrm{~m} \AA$ (continuum point) from a fixed wavelength position close to the nominal line centre, with a cadence of $\sim 33 \mathrm{~s}$ and a spectral resolution of $85 \mathrm{~m} \AA$. See Smitha \& Solanki (2017) for details about the properties of the Fe I 5250.2 $\AA$ line. SUNRISE observed the North polar region of the Sun with IMaX, field of view (FOV) being $50^{\prime \prime} \times 50^{\prime \prime}$.

These data were reduced using the standard IMaX reduction routines described in Martínez Pillet et al. (2011). After applying the dark current subtraction and flat field correction, the interference fringes and the effect of dust particles in optical elements were removed using a customised Fourier filter. The smearing of the IMaX data due to the spatial point spread function (PSF) was corrected using the phase-diversity (PD) technique (Gonsalves et al. 1979; Löfdahl \& Scharmer 1994). The $\mathrm{PD}$ restoration, done in the Fourier domain, requires an apodisation of the FOV, that reduces the IMaX FOV by roughly 30 pixels on all sides to $48^{\prime \prime} \times 48^{\prime \prime}$ (Martínez Pillet et al. 2011). Then the data were demodulated using the inferred polarimetric demodulation matrix. Furthermore, cross-talk removal and a simple correction for global stray-light was done by subtracting $12 \%$ of the spatial mean of each of the four Stokes profiles from the corresponding individual Stokes profiles, that is to say the Stokes profiles for individual spatial locations (Kahil et al. 2017).

The PD reconstructed IMaX data have a spatial resolution of $\sim 0$." $15-0$." 18 (the plate scale is 0. . $^{\prime} 055 \mathrm{pixel}^{-1}$ ) in all Stokes parameters. The noise level $(\sigma)$ for PD restored data is of the order of $\sim 3.9 \times 10^{-3}$ in Stokes $Q, U$ and $V$ signals. To minimise the influence of noise on our inferences, we restrict our analysis to those pixels whose signal strength in one of the three polarised Stokes parameters exceeds $2 \sigma$. These PD reconstructed data are used for the analysis where the high spatial resolution of SUNRISE is required, these data are not binned. The unrestored data are used for studies profiting from high magnetic sensitivity: we achieved a noise level in Stokes $Q, U$ and $V$ of $\sim 8.4 \times 10^{-4}$ by binning the unrestored data $3 \times 3$ to match the pixel size of Hinode SOT/SP $\left(0\right.$ ". $\left.16 \mathrm{pixel}^{-1}\right)$. Figure 1 shows a continuum intensity image in the top panel and the corresponding Stokes $V$ image in the bottom panel from the PD reconstructed data, without the apodisation edges introduced due to the PD restoration. In Fig. 1, both the continuum and Stokes $V$ intensity has been cut-off at about 20 pixels before the limb, and limb darkening has also been removed. This was done by fitting a fifth order polynomial in $\mu$ to the continuum intensity averaged over bins of constant $\mu$ values and using it to normalise the observations.

Our focus lies not only on the large magnetic patches (marked by red boxes in Fig. 1) but also on smaller magnetic concentrations distributed over the FOV, whose presence is well visible in the Stokes $V$ image in the bottom panel of Fig. 1. In our analysis, we use the following patch selection criteria (PSC): firstly we use PD restored data and retain only those pixels whose signal strength in one of the three polarised Stokes 


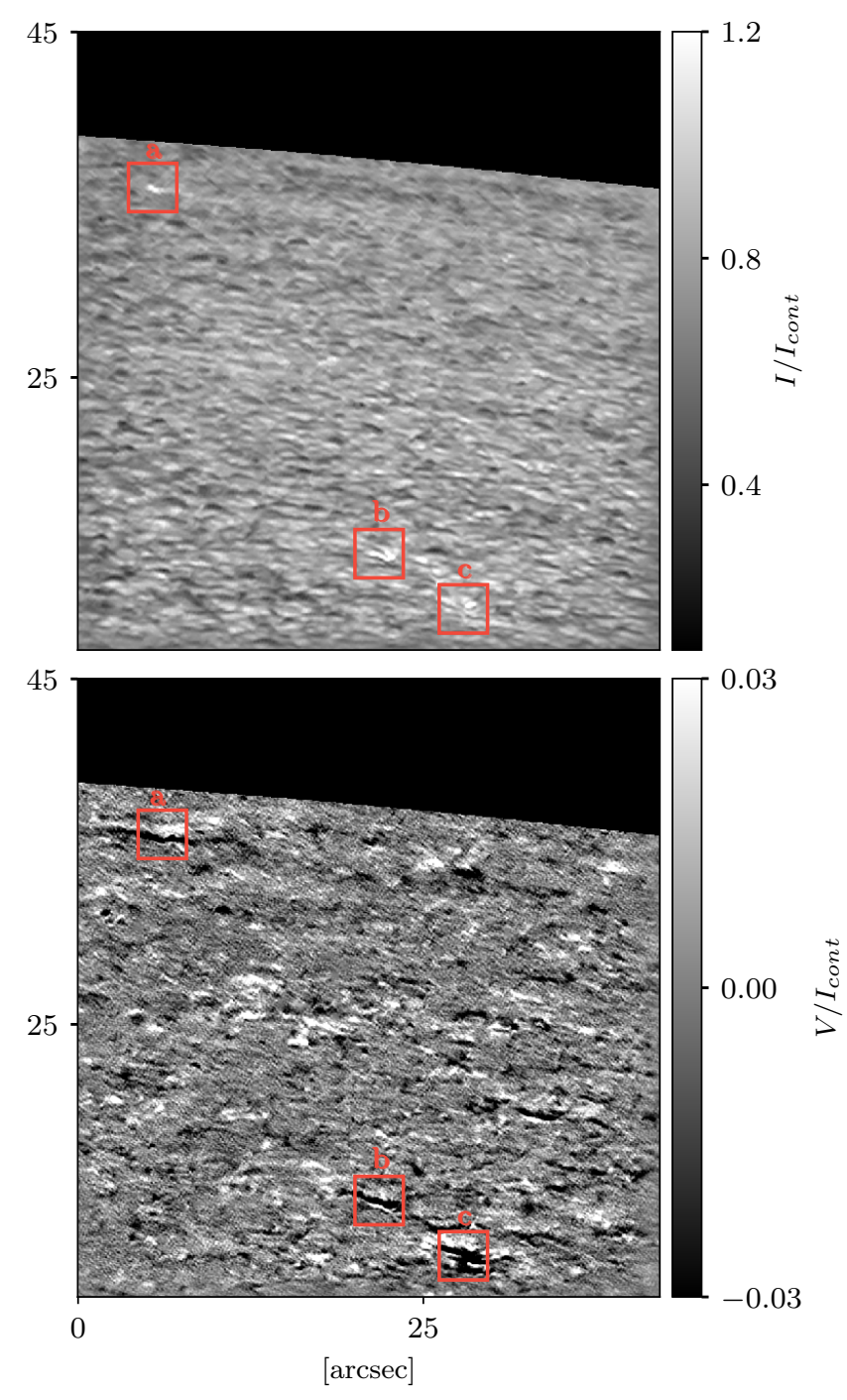

Fig. 1. Continuum intensity image (top) and Stokes $V$ (bottom) recorded by IMaX, showing nearly the entire FOV, except the apodisation edges (see text). Red boxes indicate large magnetic patches, which we look at closely in Sect. 4. The heliographic coordinates of the central pixel in both images are $85^{\circ} \mathrm{N}$ and $75^{\circ} \mathrm{W}$.

parameters exceeds $2 \sigma$, additionally every patch contains at least one pixel that has signal above $4 \sigma$ in either linear or circular polarisation Stokes parameters. Then we make sure that every patch has a minimum size of 4 contiguous pixels. To reiterate, only groups of pixels meeting all three of the aforementioned noise and size thresholds are used in the subsequent analysis. Thus for the patches studied here, the minimum area of the patches is $\sim 0.005 \mathrm{Mm}^{2}$ and the minimum flux per patch is $\sim 3.7 \times 10^{15} \mathrm{Mx}$. This value is $\sim 4.1$ times larger than the minimum flux of $9 \times 10^{14} \mathrm{Mx}$ detected by Anusha et al. (2017). The difference can be attributed to the effects of foreshortening and the inclination of the magnetic field vector with respect to the line-of-sight.

\section{Setup of inversions}

In order to interpret the Stokes profiles measured by IMaX, we use the SPINOR (Frutiger et al. 2000) code to infer a possible atmosphere from the spectropolarimetric measurements. SPINOR is a $1 \mathrm{D}$, plane-parallel inversion code solving the set of polarised radiative transfer equations (RTE) assuming a stratified solar atmosphere under local thermodynamic equilibrium, wherein parameters characterising the atmosphere are allowed to vary with optical depth. It uses the STOPRO routines for the solution of the RTE (Solanki 1987). Lagg et al. (2010) have demonstrated that the high spatial resolution of SUNRISE data allows detecting $\mathrm{kG}$ fields in the quiet-Sun at disc centre without the necessity of introducing a filling factor. We therefore use a single component atmosphere, i.e., without a magnetic filling factor, for the inversions of the PD-reconstructed polar data. For comparison, we also invert the unrestored data with a two component atmosphere (one magnetic and another non-magnetic stray light component, weighted with the magnetic filling factor). Given the limited spectral sampling of IMaX, we resorted to a relatively simple atmosphere with a total of eight free parameters: three to describe the temperature stratification at distinct optical depths, height independent magnetic field vector (strength, $B$, inclination, $\gamma$, and azimuth, $\phi$ ), microturbulent velocity $\xi_{\text {mic }}$, and lineof-sight velocity, $v_{\text {LOS }}$. As described in Lagg et al. (2010), neighbouring spectral lines might be shifted to the IMaX wavelength in the presence of large line of sight velocities. Accordingly, we also include the Co I line at 5250.0 $\AA$ and the Fe I line at 5250.6 in our analysis with the same atmospheric model as used by the IMaX line. The large and varying heliocentric angle in the FOV implies that the radiation we receive, originates from progressively higher layers in the atmosphere, as we move away from the disc centre towards the limb. To take this into account, we placed the three nodes describing the temperature stratification at three distinct optical depths for each pixel, based on the Eddington-Barbier (EB) relation (Hubeny \& Mihalas 2014). This relation states that, assuming the source function to be a linear function of optical depth, $S(\tau)=a+b \tau$ ( $\tau$ stands for optical depth), the general formal solution of the RTE for the emergent radiation at $\tau=0$ can be written as:

$I(\tau=0, \mu)=a+b \mu=S(\tau=\mu)$.

Herein $\mu$ is the cosine of the heliocentric angle. Although in reality the source function does not exhibit a linear dependence on optical depth, the EB relation is a very good approximation in the vicinity of $\tau=1$. Therefore we placed the three nodes for temperature at $\log \tau=[\log \mu, \log \mu-1, \log \mu-2.75]$.

To gain confidence in the reliability of the EBapproximation, we performed an additional inversion with a different approach by emulating the limb observations as disc centre observations. We placed the nodes for temperature stratification at $\log \tau=0,-1$ and -2.75 , as one would for disc centre observations. However, to account for the increase in the geometric height of the $\tau=1$ surface, we changed the value of the gravitational constant of the Sun for each pixel within SPINOR from $g=27400 \mathrm{~cm} \mathrm{~s}^{-2}$ to $g^{\prime}=g \cdot \mu$. This reduced gravity artificially expands the solar atmosphere in a similar way as the path length of an inclined line-of-sight passing through the solar atmosphere is expanded. Figure 2 is an illustration of the comparison between the two inversions: the EB approach and the emulated disc centre (EDC) approach. The $y$-axis shows the difference between the two approaches in retrieving the atmospheric parameters. To some extent this difference can be explained by the dissimilar numerical setups of the two approaches, resulting in an altered convergence path during the minimisation process. Combined with the polarimetric noise, this produces differences in the retrieval 


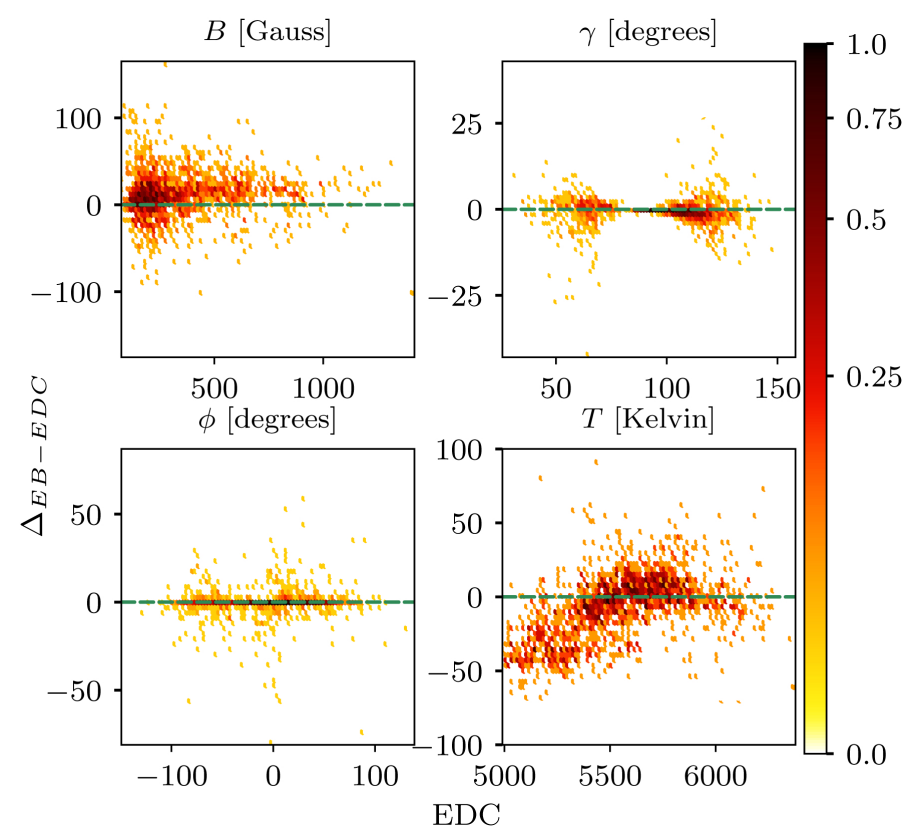

Fig. 2. Comparison of two different approaches of inverting IMaX data away from disc centre. Eddington-Barbier (EB) vs. Emulated disc centre (EDC) approach for four free parameters of the inversions: strength, inclination and azimuth of the magnetic field vector and temperature. The $y$-axis is the difference in the retrieved parameters from EB and EDC. The $x$-axis is the value of the parameter from EDC. Temperature is plotted for $\log \tau=\log \mu$. The colour scale represents normalised number density.

of the magnetic field parameters. The scatter in the magnetic field strength retrieval lies within the typical range of errors for polarimetric measurements with the noise level of our data (see Sect. 2), however it shows a systematic offset of $\sim 15 \mathrm{G}$. The determination of temperature at $\log \tau=0$ is based mainly on the continuum intensity, and therefore should not be significantly affected by polarimetric noise. For this reason, the difference in temperature retrieved by the two methods, despite being small, must be systematic. This systematic difference is clearly visible in Fig. 2, showing the largest deviation at low temperatures, i.e., pixels close to the limb. We attribute systematic differences, in both the temperature and magnetic field strength retrievals, to the continuum normalisation of the observations: the EDC approach requires the continuum to be normalised by the quiet-Sun intensity at disc centre by assuming a theoretical limb darkening profile. On the other hand for the EB approach, the continuum is normalised by the quiet-Sun intensity directly from the observations, without any extrapolation. However, as the magnetic field parameters lie within the typical scatter for measurements with the given noise level, we conclude that the magnetic field determination is robust and not dependent on using one of the two methods.

We invert all four Stokes profiles for both, the PD restored (i.e. corrected for smearing due to the spatial PSF) data and also the non-reconstructed data (spatially binned to match the resolution to Hinode). We invert the entire FOV, each pixel separately, but only look at those magnetic patches meeting the PSC mentioned in Sect. 2. We then use the PSC to segment the magnetic field maps obtained from inversions to obtain quantities such as total flux per patch (by summing over all pixels within a patch) that we then use in our analysis. To take into account the spectral resolution of the IMaX instrument as given by the filter band-width of the Fabry-Pérot etalon, the synthetic spectra are convolved with the spectral PSF of IMaX, approximated with a Gaussian with a full width at half-maximum of $85 \mathrm{~m} \AA$ (Martínez Pillet et al. 2011). We employ a form of spatial regularisation in the inversion process, in order to avoid noisy maps for the inverted atmospheric parameters. At the end of an inversion interim run (consisting of fifteen iterations of the Levenberg-Marquardt non-linear least-squares minimisation), various smoothing filters are applied to the maps of the retrieved atmospheric parameters at the respective node positions. These smoothed maps of atmospheric parameters are then used as an initial guess for the next inversion cycle. This procedure is repeated until the value of the merit function $\left(\chi^{2}\right)$ does not change significantly anymore. $\chi^{2}$ is defined by the squared differences between the observed and the fitted Stokes profiles multiplied a pre-defined weighting function (with different weights assigned to the Stokes parameters).

In a last step, we transform the line-of-sight magnetic field vector to the local solar coordinate system by employing the approach of Tsuneta et al. (2008b, described in Sect. 3.2 of their paper). Vertical fields are defined as fields with local inclination angles smaller than $25^{\circ}$ and horizontal when the local inclination is larger than $65^{\circ}$. Fields with a local inclination between $25^{\circ}$ and $65^{\circ}$ are not included in the analysis. We thus make an assumption that the magnetic field vector is either nearly vertical or nearly horizontal to the local solar surface. This approach is followed to make our results comparable to those of Tsuneta et al. (2008b).

\section{Results}

We present here the results of the inversions using the EB approach (Sect. 3) of IMaX data from two consecutive observation runs. We inverted the entire usable FOV. We included, however, only those pixels that meet our size and noise thresholds (PSC) in our subsequent analysis. In Fig. 3, we compare the distributions of vertical and horizontal fields (with respect to the local reference frame) inferred from reconstructed SUNRISE data with the distributions inferred from Hinode data, as reported by Tsuneta et al. (2008b). We want to emphasise here that the $\mathrm{kG}$ field strengths in the Hinode data are only retrieved after applying a filling factor, whereas the $\mathrm{IMaX}$ data deliver these values directly without taking recourse to such a measure.

In both observing runs, the vertical magnetic fields dominate the strong field regime and the horizontal fields are ubiquitous for field strengths below $\sim 300 \mathrm{G}$. The distributions for the vertical fields (red curve) from both data sets are in reasonably good agreement, in that both showing $\mathrm{kG}$ field strengths. There are, however, a few differences: Firstly, in the case of SUNRISE data the peak for the vertical fields is at $\sim 170 \mathrm{G}$, $20 \mathrm{G}$ higher than for Hinode. In the case of horizontal fields, the peak in our distribution is at $148 \mathrm{G}, 25 \mathrm{G}$ higher than for the Hinode data. Secondly, Hinode shows more strong vertical fields, i.e. fields stronger than $600 \mathrm{G}$, compared to SunRISE. Thus, Tsuneta et al. (2008b) in their study report numerous $\mathrm{kG}$ magnetic concentrations including larger patches. Additionally, we do not retrieve any horizontal fields higher than $\sim 800 \mathrm{G}$, whereas Tsuneta et al. (2008b) do report a significant number of horizontal $\mathrm{kG}$ field structures.

There are a number of reasons for these differences. The first difference reflects the different noise levels in the two 


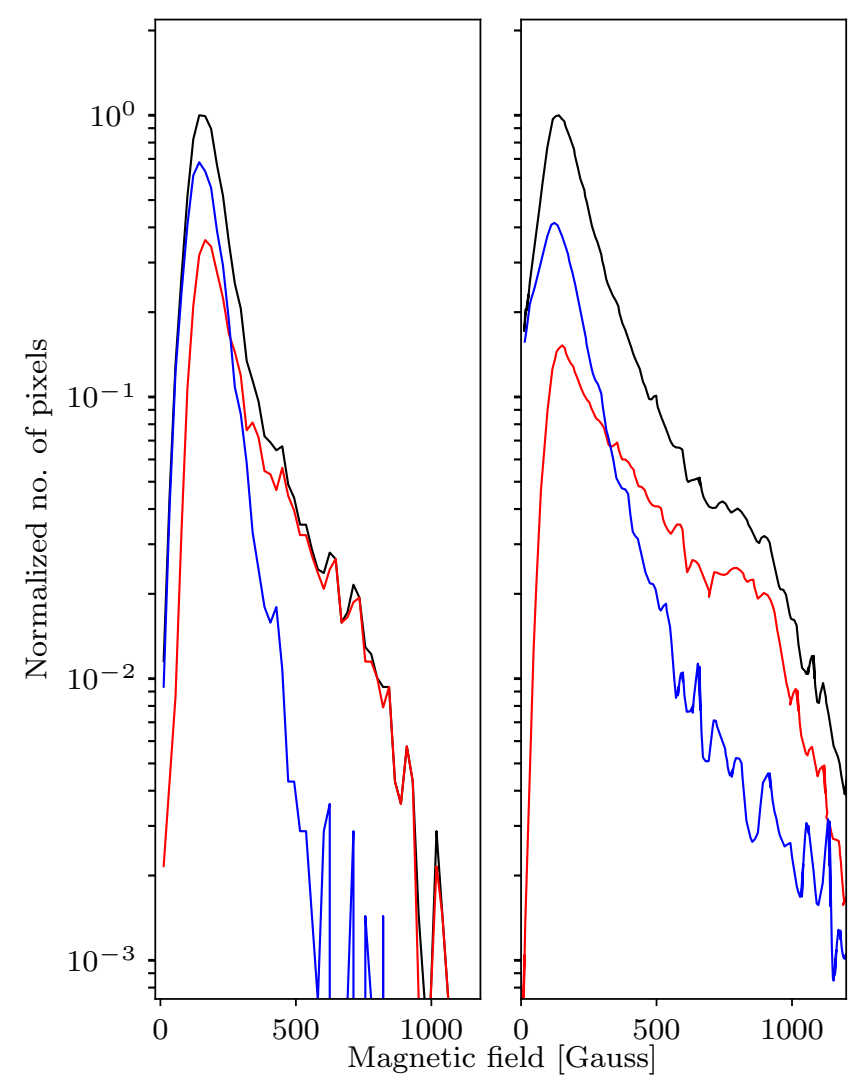

Fig. 3. Histogram of magnetic field strength, retrieved from a single component inversion of PD restored data (left) only for the pixels meeting the noise and size thresholds described in Sect. 2. A similar histogram, digitised from Tsuneta et al. (2008b), is shown on the right for comparison. The black lines show the distributions for all pixels, the red lines represent pixels with fields vertical to the local surface and the blue lines indicate pixels with fields horizontal to the surface. In both cases the number of pixels is normalised to the maximum number of pixels in the black line.

datasets. The steep drop off in the number of pixels at lower field strengths, for both SUNRISE and Hinode, is in a regime dominated by noise. Since the noise levels for IMaX observations are higher in comparison, the peak for horizontal fields is at slightly higher field values. Secondly, the Hinode/SP scan covered a significantly larger field of view, that is, 327.52" (east-west) by 163.84" (north-south along the line of sight) as compared to SUNRISE. Therefore, it managed to catch a larger part of the polar scene, including the rarer larger magnetic structures with the strongest fields. The main reason for the difference is that a filling factor was used when analysing the Hinode data, but not for the SUNRISE data. Despite the high resolution of the SUNRISE mission, there are still unresolved magnetic structures, and without using a filling factor, the inversion returns somewhat weaker and more horizontal fields.

In a next step, we look at the morphology of the largest magnetic patches in our observations and then analyse them in the context of all magnetic patches in our FOV. There are three relatively large magnetic patches embedded with bright faculae in the FOV, marked by red boxes in Fig. 1. Figure 4 shows a closer view of two of these patches (b and $c$ in Fig. 1). The patches contain contiguous pixels of high magnetic field strength, with a maximum value around $1350 \mathrm{G}$. These magnetic structures are accompanied by a facular brightening in

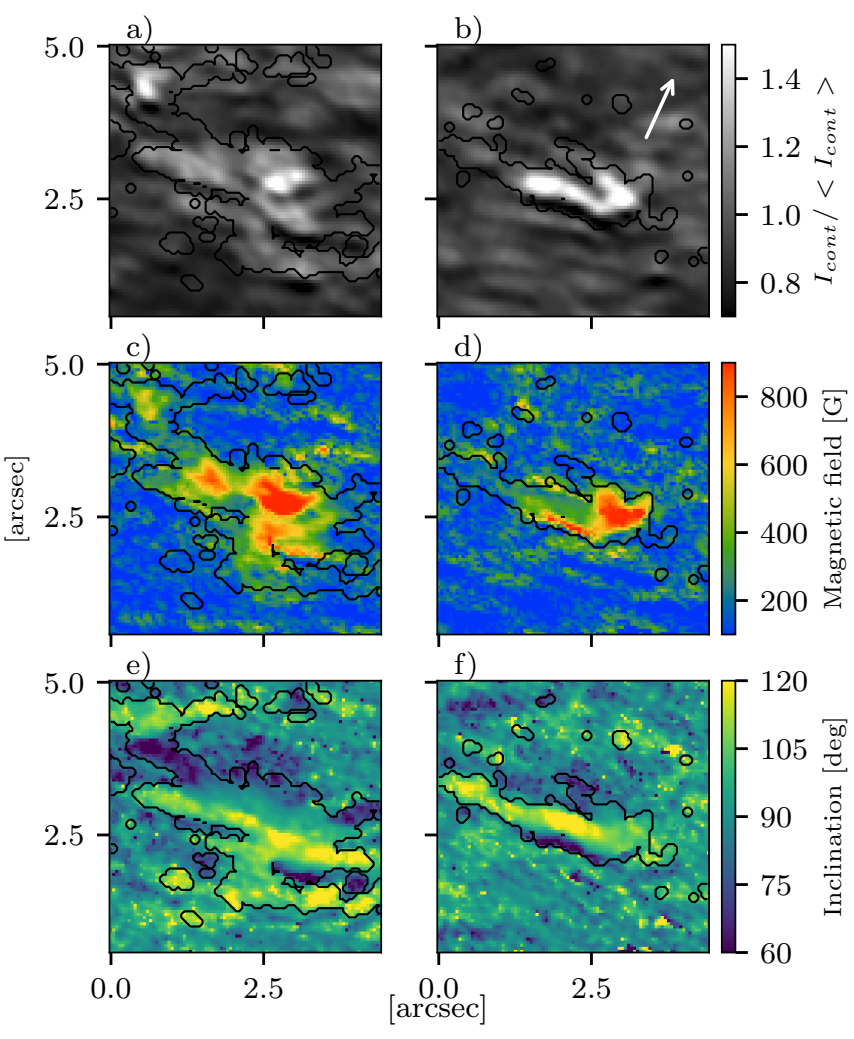

Fig. 4. Two examples of polar faculae: continuum intensity ( $a$ and $b$ ), magnetic flux in Maxwell ( $c$ and $d$ ) and line-of-sight inclination in degrees $(e$ and $f$ ) for these patches. The black contour lines enclose pixels which meet the noise and size thresholds described in Sect. 2. The white arrow indicates the limbward direction.

the continuum intensity images, as can be seen in panels a and $b$ of Fig. 4. Dark, narrow lanes in the continuum intensity are found to lie on the disc-centre side of these brightenings, as earlier pointed out by Keller et al. (2004), Carlsson et al. (2004) and Hirzberger \& Wiehr (2005). Although present in both features, these dark lanes are more evident in panel $b$ of Fig. 4. We also note that the maxima of the magnetic field are slightly shifted with respect to the continuum brightening towards the disc centre by $\sim 0.5$, in agreement with the findings of Quintero Noda et al. (2016). This shift, and the bright and dark features can be explained in the context of the 'brightwall' effect of Spruit $(1976,1977)$ and is discussed in more detail in the next section. The corresponding line-of-sight inclination angle of the magnetic field vectors within these patches is indicative of a magnetic field structure that expands with height (with the field pointing into the Sun in these particular cases).

In Fig. 1 (bottom panel), there are numerous concentrations with a significant Stokes $V$ signal outside of the large magnetic patches (red boxes from Fig. 1) scattered over FOV, ranging from the smallest (4 contiguous pixels based on the size criteria) to the large magnetic concentrations with embedded faculae. Figure 5 shows the distribution of peak field strength of patches vs. their area (panel a) and versus their total flux (panel b). We find that the peak field strength within a patch increases as the total flux and also the area of the patch increases. In panel $b$ the field strength increases sharply for smaller patches (up to $8 \times 10^{14} \mathrm{~cm}^{2}$ in size) with total flux of up to $\sim 10^{16} \mathrm{Mx}$, and above this value the increase is relatively gradual. The initial steep increase is very likely due to the smallest patches with total 

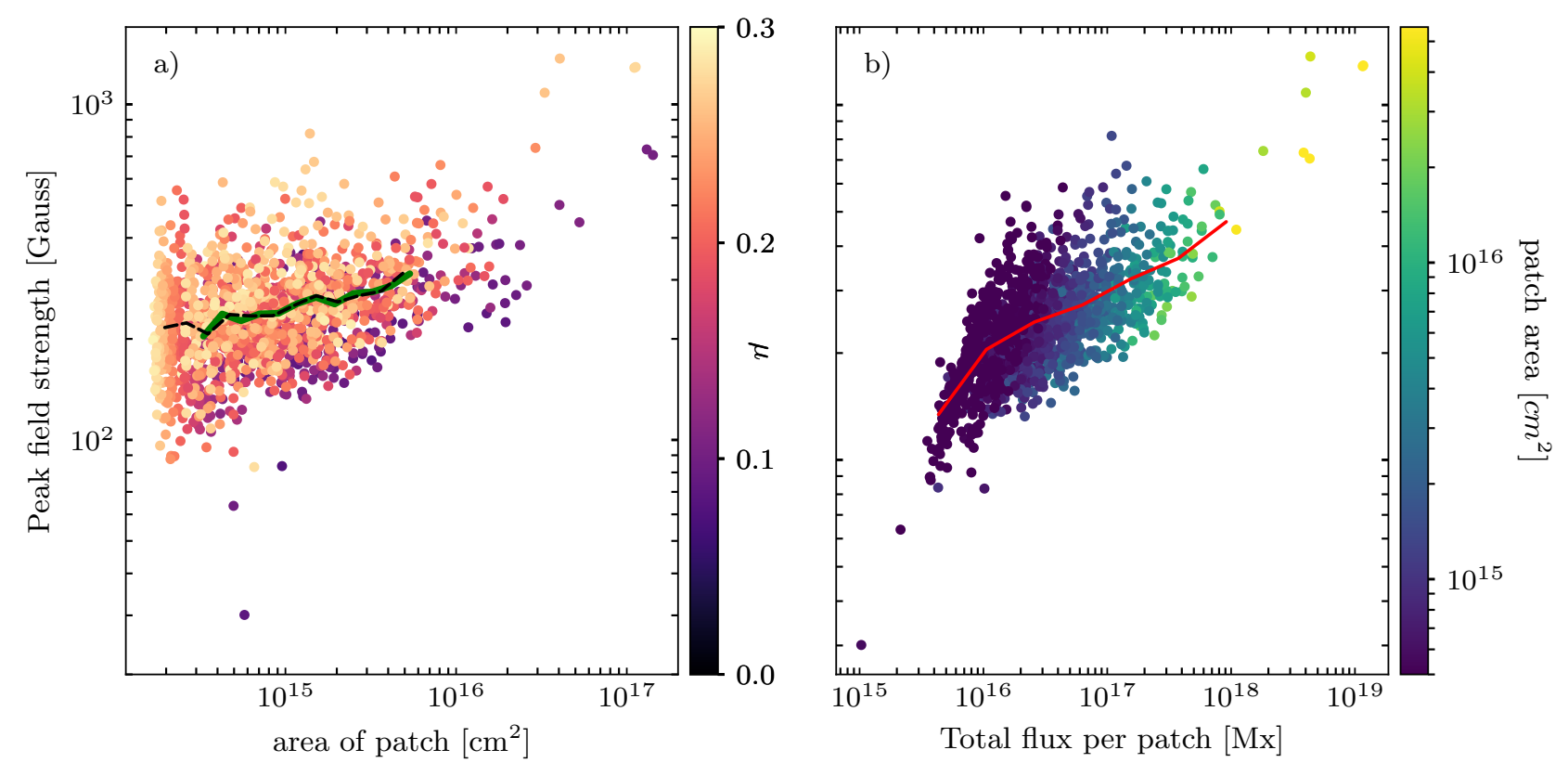

Fig. 5. Peak magnetic field within a patch versus area of the patch (panel $a$ ) and versus total flux per patch (panel $b$ ). Each data point represents a magnetic patch, with the colour in panel a showing its $\mu$ value and in panel $b$ its area, corrected for the heliocentric position of the patch. In panel $a$, the green curve shows the average value of the data points in logarithmic spaced bins for patches with $\mu \leq 0.17$ and the dotted black curve is for patches with $\mu \geq 0.17$. The red curve in panel $b$ shows the average value of all data points in logarithmically spaced bins. The plot contains all patches from two consecutive observing runs of the full Stokes vector recorded by IMaX, which meet the PSC described in Sect. 2.

flux $\leq 10^{16} \mathrm{Mx}$ not being fully resolved, thus we retrieve weaker field strengths without introducing a filling factor. In panel a we find the smaller patches to show a larger scatter in magnetic field strength, which gradually decreases with increasing size as well total flux (panel b). Since the black and green curves in panel a are almost falling on top of each other we conclude that there is no dependence on the $\mu$ value of the patch.

We also looked at the distribution of patch areas (black curve) and their polarity distribution (red and blue curves) in our FOV in Fig. 6. To determine the polarity of a patch we summed up the Stokes $V$ signal at one filter position for all pixels that are classified as vertical according to the method described in Sect. 3. The sign of this sum determines the polarity of the patch. This polarity determination, found to be the same for all 4 filter positions within the spectral line, was only applied to those patches that have vertical fields (the polarity of the patches with only horizontal fields was not determined i.e. they are not included in the red and blue curves). In 2009, the majority polarity of the North polar region was negative. We found patches of positive polarity up to an area of $\sim 2.5 \times 10^{16} \mathrm{~cm}^{2}$ and patches of negative polarity up to an area of $\sim 1.5 \times 10^{17} \mathrm{~cm}^{2}$. The maximum net flux per patch for positive polarity is $\sim 2.3 \times 10^{17} \mathrm{Mx}$ and for the negative polarity it is $\sim 1.2 \times 10^{19} \mathrm{Mx}$. Above $\sim 2.3 \times 10^{17} \mathrm{Mx}$, patches are unipolar and negative, in accordance with the dominant polarity of the north polar region during this phase of the solar cycle. This indicates that the large $\mathrm{kG}$ flux concentrations in the polar region are responsible for maintaining the major polarity of the polar region, in agreement with the findings of Shiota et al. (2012). For smaller patches, we found the minority (positive) polarity patches to dominate. This deviation from the polarity balance is unexpected and possibly results from instrumental and viewing geometry effects: Some patches with only horizontal fields have not been included in the analysis, the disambiguation might introduce a systematic effect and the calibration offset of IMaX zero level cannot be determined to the required accuracy level. In our FOV, the total flux of all positive polarity patches is $\sim 8 \times 10^{18} \mathrm{Mx}$ and for the negative (majority) polarity patches is $\sim 1.8 \times 10^{19} \mathrm{Mx}$. The latter is reduced to a value of $\sim 3.3 \times 10^{18} \mathrm{Mx}$ when excluding the unipolar (negative) patches larger than $2.5 \times 10^{16} \mathrm{~cm}^{2}$ in size.

In Fig. 7, we analyse the dependence of the temperature on the magnetic field strength. Each data point in this figure represents a pixel in the FOV; and the data points are colour-coded according to their heliocentric angle ( $\mu$-value). Three distinct populations can be identified, highlighted in the three panels of this figure: One population includes the points (solid circles; see Fig. 7a), which belong to magnetic structures outside the large magnetic patches. This population is characterised by an increase in temperature with increasing $\mu$-value and a maximum deduced field strength of $\sim 500 \mathrm{G}$. A second population is formed by the data points (solid asterisks; see Fig. 7b) associated with the patch closer to the limb (box a in Fig. 1) with $\mu$-values between $\sim 0.1-0.13$, which are hotter (brighter) and harbour higher intrinsic field strengths $(>500 \mathrm{G})$ compared to the pixels with similar $\mu$-values lying outside of the patch. The third population contains the data points (solid triangles; see Fig. 7c) which belong to the two large patches closer to the disc centre (box b and $\mathrm{c}$ in Fig. 1) with $\mu$-values greater than 0.25 . This population shows the highest temperature in the entire FOV, and also the maximum intrinsic field strength. There are pixels with temperatures higher than $6000 \mathrm{~K}$, but with field strengths of $\sim 500 \mathrm{G}$ and pixels with temperatures less than $5800 \mathrm{~K}$ but with $\mathrm{kG}$ magnetic field values. This behaviour is a result of the shift in the maxima of the magnetic field with respect to the continuum brightening seen in Fig. 4.

\section{Discussion}

The high spatial resolution of the IMaX observations together with the PD reconstruction (to correct for the spatial PSF) made it possible to invert the observations without a filling factor. We 


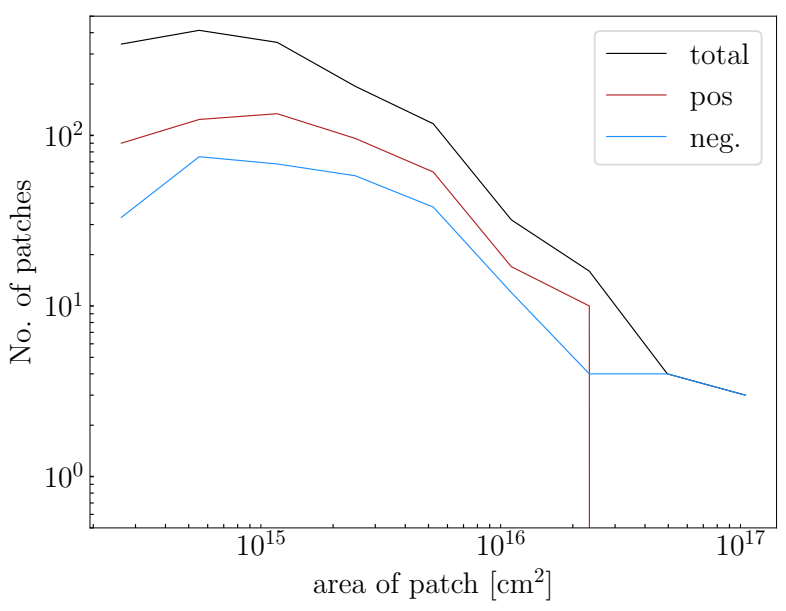

Fig. 6. Histogram of the area of the magnetic patches in the FOV (black curve) as a function of patch area. The blue (red) curve is for patches with a negative (positive) polarity. The dominant polarity of the north polar region in 2009 was negative.

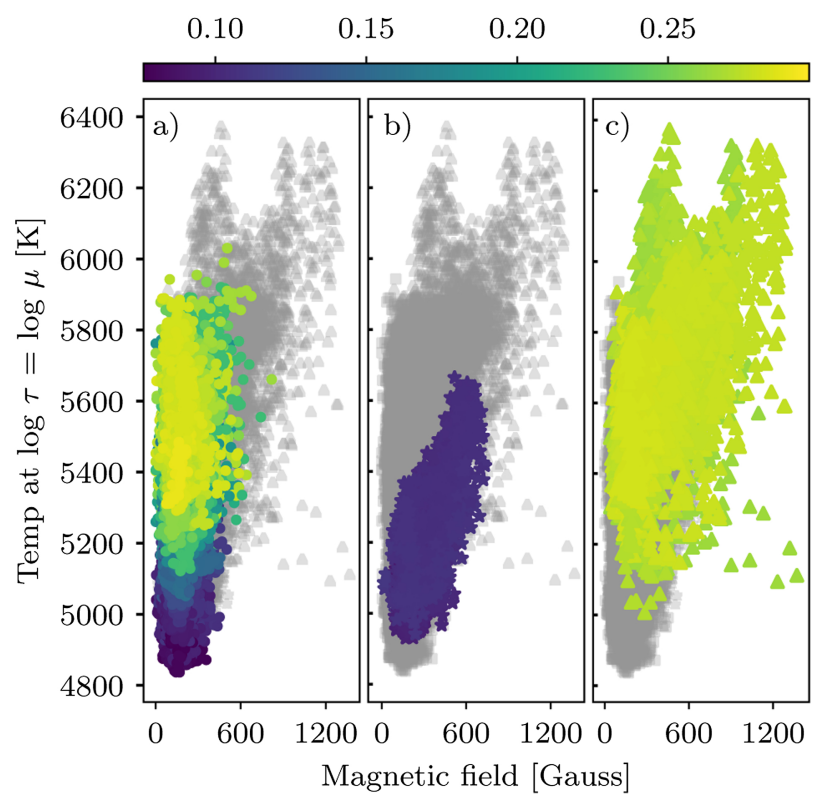

Fig. 7. Scatter plot of magnetic field strength versus temperature. All three panels show the same pixels but the different populations (see text) are highlighted by colour coding with $\mu$. The data points in the figure are pixels in the FOV which meet the noise thresholds. Pixels from large magnetic patches with embedded polar faculae (panels $b$ and $c$ ) are denoted with asterisks and solid triangles. The plot has data points from two consecutive observing runs of IMaX.

retrieved $\mathrm{kG}$ field strengths in the larger magnetic patches within the FOV. Tsuneta et al. (2008b) had resorted to a two-component (with magnetic and straylight components) atmosphere inversion, which was necessary because of the lower spatial resolution of the Hinode telescope. To test the need for a filling factor to invert data at the spatial resolution of Hinode, we performed additional experiments. In these tests, we analysed $\mathrm{IMaX}$ data not restored using the PD technique. We binned these unrestored Stokes images $3 \times 3$, to make the spatial resolution comparable to that of Hinode. We then inverted these unrestored data, with the same inversion setup as described in Sect. 3. The inversion of these spatially-smeared and binned IMaX data did not result in the retrieval of $\mathrm{kG}$ fields in the large magnetic concentrations.

We see evidence of facular brightening in Fig. 4 (panel a and $b$ ), where the locations of the strongest magnetic fields are shifted (towards disc centre) with respect to the bright regions in the continuum intensity. Additionally we also see dark, narrow lanes towards the disc-centre side adjacent to the bright faculae. These features agree very well with the extension of the "bright-wall" model of Spruit $(1976,1977)$ proposed by Keller et al. (2004) and Carlsson et al. (2004). The magnetic concentrations are in an approximate pressure balance with the surrounding non-magnetic plasma of the same geometric height. The increased magnetic pressure in these concentrations results in their evacuation. The opacity decreases locally in the less dense interiors of the magnetic features, so that the $\tau=1$ surface is depressed. Consequently, when seen from an angle (corresponding to a vertically oriented magnetic feature near the limb), most of the continuum radiation emerges from the interface of the flux concentration and the limbward granule, explaining the slight shift in the location of the magnetic field maxima and the continuum brightening. The appearance of the dark lanes in front of bright faculae was thoroughly investigated by Keller et al. (2004) and Carlsson et al. (2004). Following Keller et al. (2004) the line of sight, in addition to being dominated by the "bright-wall" of the limbward granule also passes through the cool, dense downflowing material at the interface of the disc-centreward granule and the flux concentration, explaining the appearance of these dark lanes.

Figure 7c highlights data points from the large magnetic concentrations (coloured solid triangles). There pixels with high temperatures and average field strengths, as well as pixels with $\mathrm{kG}$ field strengths and average temperatures can be identified. This is yet another manifestation of the shift in the intensity maxima and magnetic field maxima, described above. Additionally, we can see from the inclination angles between the lineof-sight and the magnetic field in panels e and $\mathrm{f}$ of Fig. 4 that the magnetic field in the large magnetic concentrations expands with height. The circular polarisation signal of these magnetic concentrations is bipolar (see Fig. 1 bottom panel), unlike the unipolar appearance at disc centre, consistent with the magnetic field fanning out with height (Pietarila et al. 2010).

In addition to the large $\mathrm{kG}$ patches discussed above, we found the polar landscape to be covered by magnetic patches of varying size. Figure 5 captures the increase of magnetic field strength of the patches with increasing magnetic flux and patch size. Smaller patches with lower total flux show a significant dispersion in field strengths with no $\mathrm{kG}$ fields. This dispersion decreases with increasing size (flux), where the largest patches with higher flux harbour $\mathrm{kG}$ fields. For smaller flux concentrations the scatter in field strengths, and the largely weak fields can be partly understood in the context of the earlier studies of Venkatakrishnan (1986), Solanki et al. (1996), Grossmann-Doerth et al. (1998), in which the evacuation of these flux concentrations by the convective collapse process (Parker 1978; Spruit 1979; Solanki 1993) is hindered by the more efficient radiative heating from the surroundings. Other factors leading to the larger scatter may be the lower signal values in the smaller features, and the greater scatter in inclination of weaker fields (for weak fields, small changes in Stokes $Q$ or $U$ correspond to large changes in inclination and/or B). Also we would like to note that we have very few large $\mathrm{kG}$ patches in our FOV to comment on their dispersion in peak field strength or area. 
We also found that in our FOV patches tend to have negative polarity above a size of $\sim 2.5 \times 10^{16} \mathrm{~cm}^{2}$ (see Fig. 6), which corresponds to a flux of $\sim 2.3 \times 10^{17} \mathrm{Mx}$, and have the same polarity as the majority polarity of the north pole in 2009 . This is close to the value reported by Shiota et al. (2012) of $10^{18} \mathrm{Mx}$. It implies that the polarity of the polar regions is maintained by large $\mathrm{kG}$ flux concentrations. Additionally, we found a polarity imbalance for smaller patches (smaller flux), which is in contrast with the findings of Pastor Yabar et al. (2020), who report a balance of polarities at smaller flux values. The lack of flux balance at small scales in our case is surprising. We have carried out ad hoc estimations of the required polarimetric zero level offset to produce such an imbalance, the results show that the imbalance cannot be accounted for by the calibration offset of the IMaX zero level. Other possible factors that could partly be responsible for differing findings of the two studies are that firstly our observations are during solar minimum, as opposed to theirs which are during solar maximum. In addition, they employ a different proxy to estimate polarity.

If we consider the size of the patches, we see that the largest magnetic patch in our distribution in Fig. 6 is 2537 pixels large. Anusha et al. (2017), in their study of small magnetic features in the quite Sun also with the IMaX instrument, had reported the largest feature size of 1585 pixels. The large size of the unipolar patches in our study is compliant with Ito et al. (2010), who find that the kG flux concentrations on the Sun's polar region are on average larger than those of the quiet Sun and, mostly due to the viewing geometry, also appear brighter.

If we look at Eq. (1), the emergent intensity samples higher layers near the limb (lower $\mu$ values), where the source function achieves smaller values, leading to lower intensity (centreto-limb variation or CLV of emergent intensity). Figure 7 captures this CLV of intensity nicely under the assumption that we are dealing with 1D atmospheres. The pixels outside of the large patches (panel a, solid circles) show progressively lower temperatures with decreasing $\mu$ values. In the same figure, pixels from the large magnetic patch at the extreme limb (panel b, solid asterisks), have weaker magnetic field and lower temperatures in comparison to the pixels from the other two large patches (panel c, solid triangles). However, we do acknowledge that we have a limited sample of only 3 such large patches. The field strength of the large magnetic concentrations decreases with height, as these structures expand due to a decrease in the surrounding gas pressure. This, coupled with the emergent radiation sampling higher layers closer to the limb, results in lower temperatures and weaker field strengths retrieved for the limbward large magnetic patch compared to the two large patches closer to the disc centre. These dependencies may well be enhanced by the finite thickness of the magnetic features. In such a case, radiative transfer effects occurring along the inclined rays lead to reduced Stokes amplitudes and also influence the line profiles in more subtle ways (Solanki et al. 1998).

\section{Summary}

In this study, we analysed IMaX observations of the North polar region of the Sun. We invoked the Eddington-Barbier relation to perform a $\mu$-dependent inversion of the spectropolarimetric data to infer the atmospheric parameters of the Sun's polar region. Given the high spatial resolution of the SUNRISE mission, we could retrieve kilo-Gauss magnetic fields in the large magnetic patches without resorting to a filling factor. This also led to a confirmation of the distribution of vertical and horizontal magnetic fields in the polar region, which was first reported by Tsuneta et al. (2008b). The large patches are unipolar (above a total flux per patch of $\sim 2.3 \times 10^{17} \mathrm{Mx}$ ) and contribute to the dominant polarity of the north polar region, which was negative at this phase of the solar cycle. In addition to the large patches housing polar faculae, we also found the polar landscape in our FOV covered with magnetic patches of varying size. These patches, ranging from $\sim 0$ "'2 (4 pixels) in size (limited by the size criteria, spatial resolution) up to the large $\mathrm{kG}$ flux concentrations (2537 pixels), exhibit increasing magnetic field strength with size and magnetic flux. These smaller patches show both magnetic polarities. It will be quite interesting to trace the evolution of patches of both polarities in the hope of uniquely identifying the mechanisms which maintain the abundance of majority polarity patches during solar minimum. Such a study is best carried out with data from a vantage point outside the ecliptic plane, such as those we expect from the SO/PHI instrument (Solanki et al. 2020) on board the Solar Orbiter mission (Müller et al. 2020).

Acknowledgements. This project has received funding from the European Research Council (ERC) under the European Union's Horizon 2020 research and innovation programme (Grant agreement No. 695075) and has been supported by the BK21 plus program through the National Research Foundation (NRF) funded by the Ministry of Education of Korea. The German contribution to Sunrise is funded by the Bundesministerium fuür Wirtschaft und Technologie through Deutsches Zentrum für Luft- und Raumfahrt e.V. (DLR), Grant No. 50 OU 0401, and by the Innovationsfond of the President of the Max Planck Society (MPG). The Spanish contribution has been funded by the Spanish MICINN under projects ESP2006-13030-C06 and AYA2009-14105-C06 (including European FEDER funds). The HAO contribution was partly funded through NASA grant NNX08AH38G. AP was funded by the International Max Planck Research School for Solar System Science at the University of Göttingen.

\section{References}

Anusha, L. S., Solanki, S. K., Hirzberger, J., \& Feller, A. 2017, A\&A, 598, A47 Barthol, P., Gandorfer, A., Solanki, S. K., et al. 2011, Sol. Phys., 268, 1 Berkefeld, T., Schmidt, W., Soltau, D., et al. 2011, Sol. Phys., 268, 103 Blanco Rodríguez, J., \& Kneer, F. 2010, A\&A, 509, A92

Blanco Rodríguez, J., Okunev, O. V., Puschmann, K. G., Kneer, F., \& SánchezAndrade Nuño, B. 2007, A\&A, 474, 251

Cameron, R., \& Schüssler, M. 2007, ApJ, 659, 801

Cameron, R., \& Schüssler, M. 2015, Science, 347, 1333

Carlsson, M., Stein, R. F., Nordlund, Å., \& Scharmer, G. B. 2004, ApJ, 610, L137

Frutiger, C., Solanki, S. K., Fligge, M., \& Bruls, J. H. M. J. 2000, A\&A, 358, 1109

Gabriel, A., Bely-Dubau, F., Tison, E., \& Wilhelm, K. 2009, ApJ, 700, 551

Gandorfer, A., Grauf, B., Barthol, P., et al. 2011, Sol. Phys., 268, 35

Gonsalves, R. A., \& Chidlaw, R. 1979, in Applications of Digital Image Processing III, ed. A. G. Tescher, Proc. SPIE, 207, 32

Grossmann-Doerth, U., Schuessler, M., \& Steiner, O. 1998, A\&A, 337, 928

Hassler, D. M., Dammasch, I. E., Lemaire, P., et al. 1999, Science, 283, 810

Hirzberger, J., \& Wiehr, E. 2005, A\&A, 438, 1059

Homann, T., Kneer, F., \& Makarov, V. I. 1997, Sol. Phys., 175, 81

Hubeny, I., \& Mihalas, D. 2014, Theory of Stellar Atmospheres (Princeton University Press)

Ito, H., Tsuneta, S., Shiota, D., Tokumaru, M., \& Fujiki, K. 2010, ApJ, 719, 131

Kahil, F., Riethmüller, T. L., \& Solanki, S. K. 2017, ApJS, 229, 12

Kaithakkal, A. J., Suematsu, Y., Kubo, M., Shiota, D., \& Tsuneta, S. 2013, ApJ, 776,122

Kaithakkal, A. J., Suematsu, Y., Kubo, M., et al. 2015, ApJ, 799, 139

Keller, C. U., Schüssler, M., Vögler, A., \& Zakharov, V. 2004, ApJ, 607, L59 
A. Prabhu et al.: The magnetic fine structure of the Sun's polar region as revealed by Sunrise

Knaack, R., Fligge, M., Solanki, S. K., \& Unruh, Y. C. 2001, A\&A, 376, 1080

Kosugi, T., Matsuzaki, K., Sakao, T., et al. 2007, Sol. Phys., 243, 3

Lagg, A., Solanki, S. K., Riethmüller, T. L., et al. 2010, ApJ, 723, L164

Linker, J. A., Caplan, R. M., Downs, C., et al. 2017, ApJ, 848, 70

Löfdahl, M. G., \& Scharmer, G. B. 1994, A\&AS, 107, 243

Martínez Pillet, V., Del Toro Iniesta, J. C., Álvarez-Herrero, A., et al. 2011, Sol. Phys., 268, 57

McComas, D. J., Barraclough, B. L., Funsten, H. O., et al. 2000, J. Geophys. Res., 105, 10419

Müller, D., St. Cyr, O. C., Zouganelis, I., et al. 2020, A\&A, 642, A1

Okunev, O. V., \& Kneer, F. 2004, A\&A, 425, 321

Parker, E. N. 1978, ApJ, 221, 368

Pastor Yabar, A., Martínez González, M. J., \& Collados, M. 2018, A\&A, 616, A46

Pastor Yabar, A., Martínez González, M. J., \& Collados, M. 2020, A\&A, 635, A 210

Petrie, G. J. D. 2015, Liv. Rev. Sol. Phys., 12, 5

Pietarila, A., Cameron, R., \& Solanki, S. K. 2010, A\&A, 518, A50

Quintero Noda, C., Suematsu, Y., Ruiz Cobo, B., Shimizu, T., \& Asensio Ramos, A. 2016, MNRAS, 460, 956

Schatten, K. H. 1993, J. Geophys. Res., 98, 18

Schatten, K. H., Scherrer, P. H., Svalgaard, L., \& Wilcox, J. M. 1978, Geophys. Res. Lett., 5, 411

Shimizu, T., Nagata, S., Tsuneta, S., et al. 2008, Sol. Phys., 249, 221
Shiota, D., Tsuneta, S., Shimojo, M., et al. 2012, AGU Fall Meeting Abstracts, SH13C

Smitha, H. N., \& Solanki, S. K. 2017, A\&A, 608, A111

Solanki, S. K. 1987, PhD Thesis, Max Planck Institute for Solar System Research, Justus-von-Liebig-Weg 3, 37351 Göttingen, Germany

Solanki, S. K. 1993, Space Sci. Rev., 63, 1

Solanki, S. K., Zufferey, D., Lin, H., Rueedi, I., \& Kuhn, J. R. 1996, A\&A, 310, L33

Solanki, S. K., Steiner, O., Buente, M., Murphy, G., \& Ploner, S. R. O. 1998, A\&A, 333, 721

Solanki, S. K., Barthol, P., Danilovic, S., et al. 2010, ApJ, 723, L127

Solanki, S. K., del Toro Iniesta, J. C., Woch, J., et al. 2020, A\&A, 642, A11

Spruit, H. C. 1976, Sol. Phys., 50, 269

Spruit, H. C. 1977, Sol. Phys., 55, 3

Spruit, H. C. 1979, Sol. Phys., 61, 363

Suematsu, Y., Tsuneta, S., Ichimoto, K., et al. 2008, Sol. Phys., 249, 197

Svalgaard, L., Duvall, T. L., Jr, \& Scherrer, P. H. 1978, Sol. Phys., 58, 225

Tsuneta, S., Ichimoto, K., Katsukawa, Y., et al. 2008a, ApJ, 688, 1374

Tsuneta, S., Ichimoto, K., Katsukawa, Y., et al. 2008b, Sol. Phys., 249, 167

Tu, C.-Y., Zhou, C., Marsch, E., et al. 2005, Science, 308, 519

Venkatakrishnan, P. 1986, Nature, 322, 156

Vieira, L. E. A., Norton, A., Dudok de Wit, T., et al. 2012, Geophys. Res. Lett., 39, L16104

Wang, Y. M. 2009, Space Sci. Rev., 144, 383

Wang, Y.-M., \& Sheeley, N. R. 2009, ApJ, 694, L11 